\title{
Existence and Lyapunov Stability of Periodic Solutions for Generalized Higher-Order Neutral Differential Equations
}

\author{
Jingli Ren, ${ }^{1}$ Wing-Sum Cheung, ${ }^{2}$ and Zhibo Cheng1 \\ ${ }^{1}$ Department of Mathematics, Zhengzhou University, Zhengzhou 450001, China \\ ${ }^{2}$ Department of Mathematics, The University of Hong Kong, Pokfulam Road, Hong Kong \\ Correspondence should be addressed to Wing-Sum Cheung, wscheung@hku.hk \\ Received 17 May 2010; Accepted 23 June 2010 \\ Academic Editor: Feliz Manuel Minhós
}

Copyright (c) 2011 Jingli Ren et al. This is an open access article distributed under the Creative Commons Attribution License, which permits unrestricted use, distribution, and reproduction in any medium, provided the original work is properly cited.

Existence and Lyapunov stability of periodic solutions for a generalized higher-order neutral differential equation are established.

\section{Introduction}

In recent years, there is a good amount of work on periodic solutions for neutral differential equations (see [1-11] and the references cited therein). For example, the following neutral differential equations

$$
\begin{gathered}
\frac{d}{d u}(u(t)-k u(t-\tau))=g_{1}(u(t))+g_{2}\left(u\left(t-\tau_{1}\right)\right)+p(t), \\
(x(t)+c x(t-r))^{\prime \prime}+f\left(x^{\prime}(t)\right)+g(x(t-\tau(t)))=p(t), \\
(x(t)-c x(t-\sigma))^{(n)}+f(x(t)) x^{\prime}(t)+g\left(\int_{-r}^{0} x(t+s) d \alpha(s)\right)=p(t)
\end{gathered}
$$

have been studied in $[1,3,8]$, respectively, and existence criteria of periodic solutions were established for these equations. Afterwards, along with intensive research on the $p$-Laplacian, 
some authors $[4,11]$ start to consider the following $p$-Laplacian neutral functional differential equations:

$$
\begin{gathered}
\left(\phi_{p}(x(t)-c x(t-\sigma))^{\prime}\right)^{\prime}+g(t, x(t-\tau(t)))=p(t), \\
\left(\phi_{p}\left((x(t)-c x(t-\sigma))^{\prime}\right)\right)+f\left(x^{\prime}(t)\right)+g(x(t-\tau(t)))=e(t),
\end{gathered}
$$

and by using topological degree theory and some analysis skills, existence results of periodic solutions for (1.2) have been presented.

In general, most of the existing results are concentrated on lower-order neutral functional differential equations, while studies on higher-order neutral functional differential equations are rather infrequent, especially on higher-order $p$-Laplacian neutral functional differential equations. In this paper, we consider the following generalized higher-order neutral functional differential equation:

$$
\left(\varphi_{p}(x(t)-c x(t-\sigma))^{(l)}\right)^{(n-l)}=F\left(t, x(t), x^{\prime}(t), \ldots, x^{(l-1)}(t)\right),
$$

where $\varphi_{p}: \mathbb{R} \rightarrow \mathbb{R}$ is given by $\varphi_{p}(s)=|s|^{p-2} s$ with $p \geq 2$ being a constant, $F$ is a continuous function defined on $\mathbb{R}^{l}$ and is periodic with respect to $t$ with period $T$, that is, $F(t, \cdot, \ldots, \cdot)=$ $F(t+T, \cdot, \ldots, \cdot), F(t, a, 0, \ldots, 0) \not \equiv 0$ for all $a \in \mathbb{R}$, and $c, \sigma$ are constants.

Since the neutral operator is divided into two cases $|c| \neq 1$ and $|c|=1$, it is natural to study the neutral differential equation separately according to these two cases. The case $|c|=1$ has been studied in [5]. Now we consider (1.3) for the case $|c| \neq 1$. So throughout this paper, we always assume that $|c| \neq 1$, and the paper is organized as follows. We first transform (1.3) into a system of first-order differential equations, and then by applying Mawhin's continuation theory and some new inequalities, we obtain sufficient conditions for the existence of periodic solutions for (1.3). The Lyapunov stability of periodic solutions for the equation will then be established. Finally, an example is given to illustrate our results.

\section{Preparation}

First, we recall two lemmas. Let $X$ and $Y$ be real Banach spaces and let $L: D(L) \subset X \rightarrow Y$ be a Fredholm operator with index zero; here $D(L)$ denotes the domain of $L$. This means that $\operatorname{Im} L$ is closed in $Y$ and $\operatorname{dim} \operatorname{Ker} L=\operatorname{dim}(Y / \operatorname{Im} L)<+\infty$. Consider supplementary subspaces $X_{1}$, $Y_{1}$ of $X, Y$, respectively, such that $X=\operatorname{Ker} L \oplus X_{1}, Y=\operatorname{Im} L \oplus Y_{1}$. Let $P: X \rightarrow \operatorname{Ker} L$ and $Q: Y \rightarrow Y_{1}$ denote the natural projections. Clearly, Ker $L \cap\left(D(L) \cap X_{1}\right)=\{0\}$ and so the restriction $L_{P}:=\left.L\right|_{D(L) \cap X_{1}}$ is invertible. Let $K$ denote the inverse of $L_{P}$.

Let $\Omega$ be an open bounded subset of $X$ with $D(L) \cap \Omega \neq \emptyset$. A map $N: \bar{\Omega} \rightarrow Y$ is said to be $L$-compact in $\bar{\Omega}$ if $Q N(\bar{\Omega})$ is bounded and the operator $K(I-Q) N: \bar{\Omega} \rightarrow X$ is compact.

Lemma 2.1 (see [12]). Suppose that $X$ and $Y$ are two Banach spaces, and suppose that $L: D(L) \subset$ $X \rightarrow Y$ is a Fredholm operator with index zero. Let $\Omega \subset X$ be an open bounded set and let $N: \bar{\Omega} \rightarrow Y$ be L-compact on $\bar{\Omega}$. Assume that the following conditions hold:

(1) $L x \neq \lambda N x$, for all $x \in \partial \Omega \cap D(L), \lambda \in(0,1)$,

(2) $N x \notin \operatorname{Im} L$, for all $x \in \partial \Omega \cap \operatorname{Ker} L$, 
(3) $\operatorname{deg}\{J Q N, \Omega \cap$ Ker $L, 0\} \neq 0$, where $J: \operatorname{Im} Q \rightarrow$ Ker $L$ is an isomorphism.

Then, the equation $L x=N x$ has a solution in $\bar{\Omega} \cap D(L)$.

Lemma 2.2 (see [13]). If $\omega \in C^{1}(\mathbb{R}, \mathbb{R})$ and $\omega(0)=\omega(T)=0$, then

$$
\int_{0}^{T}|\omega(t)|^{p} d t \leq\left(\frac{T}{\pi_{p}}\right)^{p} \int_{0}^{T}\left|\omega^{\prime}(t)\right|^{p} d t
$$

where $p$ is a fixed real number with $p>1$ and

$$
\pi_{p}=2 \int_{0}^{(p-1) / p} \frac{d s}{\left(1-s^{p} /(p-1)\right)^{1 / p}}=\frac{2 \pi(p-1)^{1 / p}}{p \sin (\pi / p)} .
$$

For the sake of convenience, throughout this paper we denote by $T$ a positive real number, and for any continuous function $u$, we write

$$
|u|_{0}:=\max _{t \in[0, T]}|u(t)|
$$

Let $A: C_{T} \rightarrow C_{T}$ be the operator on $C_{T}:=\{x \in C(\mathbb{R}, \mathbb{R}): x(t+T)=x(t)$ for all $t \in \mathbb{R}\}$ given by

$$
(A x)(t):=x(t)-c x(t-\sigma), \quad \forall x \in C_{T}, t \in \mathbb{R} .
$$

Lemma 2.3. The operator $A$ has a continuous inverse $A^{-1}$ on $C_{T}$ satisfying the following:

(1) $\left[A^{-1} f\right](t)= \begin{cases}f(t)+\sum_{j=1}^{\infty} c^{j} f(t-j \sigma), & \text { for }|c|<1, \forall f \in C_{T}, \\ -\frac{f(t+\sigma)}{c}-\sum_{j=1}^{\infty} \frac{1}{c^{j+1}} f(t+(j+1) \sigma), & \text { for }|c|>1, \forall f \in C_{T} ，\end{cases}$

(2) $\left|\left[A^{-1} f\right](t)\right| \leq \frac{|f|_{0}}{|1-| c||}, \quad \forall f \in C_{T}$,

(3) $\int_{0}^{T}\left|\left[A^{-1} f\right](t)\right| d t \leq \frac{1}{|1-| c||} \int_{0}^{T}|f(t)| d t, \quad \forall f \in C_{T}$.

Remark 2.4. This lemma is basically proved in $[3,10]$. For the convenience of the readers, we present a detailed proof here as follows.

Proof. We split it into the following two cases.

Case $1(|c|<1)$. Define an operator $B: C_{T} \rightarrow C_{T}$ by

$$
(B x)(t):=c x(t-\sigma), \quad \forall x \in C_{T}, t \in \mathbb{R} .
$$


Clearly, $B^{j} x(t)=c^{j} x(t-j \sigma)$ and $A=I-B$. Note also that $\|B\|<|c|<1$. Therefore, $A$ has a continuous inverse $A^{-1}: C_{T} \rightarrow C_{T}$ with $A^{-1}=(I-B)^{-1}=\sum_{j=0}^{\infty} B^{j}$; here $B^{0} x(t):=x(t)$. Hence,

$$
\left[A^{-1} f\right](t)=\sum_{j=0}^{\infty}\left[B^{j} f\right](t)=f(t)+\sum_{j=1}^{\infty} c^{j} f(t-j \sigma)
$$

and so

$$
\begin{gathered}
\left|\left[A^{-1} f(t)\right]\right|=\left|\sum_{j=0}^{\infty}\left[B^{j} f\right](t)\right|=\left|\sum_{j=0}^{\infty} c^{j} f(t-j \sigma)\right| \leq \frac{|f|_{0}}{1-|c|}, \\
\int_{0}^{T}\left|\left[A^{-1} f\right](t)\right| d t \leq \sum_{j=0}^{\infty} \int_{0}^{T}\left|\left(B^{j} f\right)(t)\right| d t=\sum_{j=0}^{\infty} \int_{0}^{T}\left|c^{j} f(t-j \sigma)\right| d t \leq \frac{1}{1-|c|} \int_{0}^{T}|f(t)| d t .
\end{gathered}
$$

Case $2(|c|>1)$. Define operators

$$
\begin{gathered}
E: C_{T} \longrightarrow C_{T}, \quad(E x)(t):=x(t)-\frac{1}{c} x(t+\sigma), \\
B_{1}: C_{T} \longrightarrow C_{T}, \quad\left(B_{1} x\right)(t):=\frac{1}{c} x(t+\sigma) .
\end{gathered}
$$

From the definition of the linear operator $B_{1}$, we have

$$
\begin{gathered}
\left(B_{1}^{j} f\right)(t)=\frac{1}{c^{j}} f(t+j \sigma), \\
\sum_{j=0}^{\infty}\left(B_{1}^{j} f\right)(t)=f(t)+\sum_{j=1}^{\infty} \frac{1}{c^{j}} f(t+j \sigma) .
\end{gathered}
$$

Since $\left\|B_{1}\right\|<1$, the operator $E$ has a bounded inverse $E^{-1}: C_{T} \rightarrow C_{T}$ with

$$
E^{-1}=\left(I-B_{1}\right)^{-1}=I+\sum_{j=1}^{\infty} B_{1}^{j}
$$

and so, for any $f \in C_{T}$,

$$
\left(E^{-1} f\right)(t)=f(t)+\sum_{j=1}^{\infty}\left(B_{1}^{j} f\right)(t)
$$

On the other hand, from $[A x](t)=x(t)-c x(t-\sigma)$, we have

$$
[A x](t)=x(t)-c x(t-\sigma)=-c\left[x(t-\sigma)-\frac{1}{c} x(t)\right] .
$$


That is,

$$
[A x](t)=-c(E x)(t-\sigma)
$$

Now, for any $f \in C_{T}$, if $x(t)$ satisfies

$$
[A x](t)=f(t)
$$

then we have

$$
-c(E x)(t-\sigma)=f(t)
$$

or

$$
(E x)(t)=-\frac{f(t+\sigma)}{c}=f_{1}(t)
$$

So, we have

$$
x(t)=\left(E^{-1} f_{1}\right)(t)=f_{1}(t)+\sum_{j=1}^{\infty} B_{1}^{j} f_{1}(t)=-\frac{f(t+\sigma)}{c}-\sum_{j=1}^{\infty} B_{1}^{j} \frac{f(t+\sigma)}{c} .
$$

So, $A^{-1}$ exists and satisfies

$$
\begin{gathered}
{\left[A^{-1} f\right](t)=-\frac{f(t+\sigma)}{c}-\sum_{j=1}^{\infty} B_{1}^{j} \frac{f(t+\sigma)}{c}=-\frac{f(t+\sigma)}{c}-\sum_{j=1}^{\infty} \frac{1}{c^{j+1}} f(t+(j+1) \sigma),} \\
\left|\left[A^{-1} f\right](t)\right|=\left|-\frac{f(t+\sigma)}{c}-\sum_{j=1}^{\infty} \frac{1}{c^{j+1}} f(t+(j+1) \sigma)\right| \leq \frac{|f|_{0}}{|c|-1} .
\end{gathered}
$$

This proves (1) and (2) of Lemma 2.3. Finally, (3) is easily verified.

By Hale's terminology [14], a solution $x(t)$ of $(1.3)$ is that $x(t) \in C^{1}(\mathbb{R}, \mathbb{R})$ such that $A x \in C^{1}(\mathbb{R}, \mathbb{R})$ and $(1.3)$ is satisfied on $\mathbb{R}$. In general, $x(t)$ does not belong to $C^{1}(\mathbb{R}, \mathbb{R})$. But we can see easily from $(A x)^{\prime}(t)=A x^{\prime}(t)$ that a solution $x(t)$ of $(1.3)$ must belong to $C^{1}(\mathbb{R}, \mathbb{R})$. Equation (1.3) is transformed into

$$
\left(\varphi_{p}\left(\left(A x^{(l)}\right)(t)\right)\right)^{(n-l)}=F\left(t, x(t), x^{\prime}(t), \ldots, x^{(l-1)}(t)\right)
$$

Lemma 2.5 (see [4]). If $p>1$, then

$$
\int_{0}^{T}\left|\left(A^{-1} f\right)(t)\right|^{p} d t \leq \frac{1}{|1-| c||^{p}} \int_{0}^{T}|f(t)|^{p} d t, \quad \forall f \in C_{T} .
$$


Now we consider (2.22). Define the conjugate index $q \in(1,2]$ by $1 / p+1 / q=1$. Introducing new variables

$$
\begin{gathered}
y_{1}(t)=x(t), \quad y_{2}(t)=x^{\prime}(t), \quad y_{3}(t)=x^{\prime \prime}(t), \ldots, y_{l}(t)=x^{(l-1)}(t), \\
y_{l+1}(t)=\varphi_{p}\left(A x^{(l)}(t)\right), \quad y_{l+2}(t)=\left(\varphi_{p}\left(A x^{(l)}(t)\right)\right)^{\prime}, \ldots, y_{n}(t)=\left(\varphi_{p}\left(A x^{(l)}(t)\right)\right)^{(n-l-1)} .
\end{gathered}
$$

Using the fact that $\varphi_{q} \circ \varphi_{p} \equiv \mathrm{id}$ and by Lemma 2.3, (1.3) can be rewritten as

$$
\begin{gathered}
y_{1}^{\prime}(t)=y_{2}(t), \\
y_{2}^{\prime}(t)=y_{3}(t), \\
\vdots \\
y_{l-1}^{\prime}(t)=y_{l}(t), \\
y_{l}^{\prime}(t)=A^{-1} \varphi_{q}\left(y_{l+1}(t)\right), \\
y_{l+1}^{\prime}(t)=y_{l+2}(t), \\
\vdots \\
y_{n-1}^{\prime}(t)=y_{n}(t), \\
y_{n}^{\prime}(t)=F\left(t, y_{1}(t), y_{2}(t), \ldots, y_{l}(t)\right) .
\end{gathered}
$$

It is clear that, if $y(t)=\left(y_{1}(t), y_{2}(t), \ldots, y_{n}(t)\right)^{\top}$ is a $T$-periodic solution to (2.25), then $y_{1}(t)$ must be a $T$-periodic solution to (1.3). Thus, the problem of finding a $T$-periodic solution for (1.3) reduces to finding one for (2.25).

Define the linear spaces

$$
X=Y=\left\{y=\left(y_{1}(\cdot), y_{2}(\cdot), \ldots, y_{n}(\cdot)\right)^{\top} \in C^{0}\left(\mathbb{R}, \mathbb{R}^{n}\right): y(t+T) \equiv y(t)\right\}
$$

with norm $\|y\|=\max \left\{\left\|y_{1}\right\|,\left\|y_{2}\right\|, \ldots,\left\|y_{n}\right\|\right\}$. Obviously, $X$ and $Y$ are Banach spaces. Define

$$
L: D(L)=\left\{y \in C^{1}\left(\mathbb{R}, \mathbb{R}^{n}\right): y(t+T)=y(t)\right\} \subset X \longrightarrow Y
$$


by

$$
L y=y^{\prime}=\left(\begin{array}{c}
y_{1}^{\prime} \\
y_{2}^{\prime} \\
\vdots \\
y_{l}^{\prime} \\
\vdots \\
y_{n}^{\prime}
\end{array}\right)
$$

Moreover, define

$$
N: X \longrightarrow Y
$$

by

$$
N y=\left(\begin{array}{c}
y_{2}(t) \\
y_{3}(t) \\
\vdots \\
A^{-1} \varphi_{q}\left(y_{l+1}(t)\right) \\
y_{l+2}(t) \\
\vdots \\
F\left(t, y_{1}(t), y_{2}(t), \ldots, y_{l}(t)\right)
\end{array}\right)
$$

Then, (2.25) can be rewritten as the abstract equation $L y=N y$. From the definition of $L$, one can easily see that Ker $L=\left\{y \in C^{1}\left(\mathbb{R}, \mathbb{R}^{n}\right): y\right.$ is constant $\} \simeq \mathbb{R}^{n}$ and $\operatorname{Im} L=\{y: y \in$ $\left.X, \int_{0}^{T} y(s) d s=0\right\}$. So, $L$ is a Fredholm operator with index zero. Let $P: X \rightarrow \operatorname{Ker} L$ and $Q: Y \rightarrow \operatorname{Im} Q$ be defined by

$$
P y=\frac{1}{T} \int_{0}^{T} y(s) d s, \quad Q y=\frac{1}{T} \int_{0}^{T} y(s) d s
$$

It is easy to see that $\operatorname{Ker} L=\operatorname{Im} Q=\mathbb{R}^{n}$. Moreover, for all $y \in Y$, if we write $y^{*}=y-Q(y)$, we have $\int_{0}^{T} y^{*}(s) d s=0$ and so $y^{*} \in \operatorname{Im} L$. This is to say $Y=\operatorname{Im} Q \oplus \operatorname{Im} L$ and $\operatorname{dim}(Y / \operatorname{Im} L)=$ $\operatorname{dim} \operatorname{Im} Q=\operatorname{dim} \operatorname{Ker} L$. So, $L$ is a Fredholm operator with index zero. Let $K$ denote the inverse of $\left.L\right|_{\text {Ker } p \cap D(L)}$, then we have

$$
[K y](t)=\left(\int_{0}^{T} G_{1}(t, s) y_{1}(s) d s, \int_{0}^{T} G_{2}(t, s) y_{2}(s) d s, \ldots, \int_{0}^{T} G_{n}(t, s) y_{n}(s) d s\right)^{\top}
$$


where

$$
G_{i}(t, s)=\left\{\begin{array}{ll}
\frac{s}{T}, & 0 \leq s<t \leq T, \\
\frac{s-T}{T}, & 0 \leq t \leq s \leq T,
\end{array} \quad i=1,2, \ldots, n\right.
$$

From (2.30) and (2.33), it is clear that $Q N$ and $K(I-Q) N$ are continuous, and $Q N(\bar{\Omega})$ is bounded, and so $K(I-Q) N(\bar{\Omega})$ is compact for any open bounded $\Omega \subset X$. Hence, $N$ is $L$ compact on $\bar{\Omega}$. For the function $y(t)=\left(y_{1}(t), y_{2}(t), \ldots, y_{n}(t)\right)^{\top}$ defined as $(2.24)$, we have the following.

Lemma 2.6. If $y(t) \in C^{1}\left(\mathbb{R}, \mathbb{R}^{n}\right)$ and $y(t+T)=y(t)$, then

$$
\int_{0}^{T}\left|y_{i}^{\prime}(t)\right|^{p} d t \leq \frac{1}{\left.|1-| c\right|^{p}}\left(\frac{T}{\pi_{p}}\right)^{p(l-i)}\left(\frac{T}{\pi_{q}}\right)^{q(n-l)} \int_{0}^{T}\left|y_{n}^{\prime}(t)\right|^{q} d t
$$

where $1 / p+1 / q=1, p \geq 2, i=1,2, \ldots, l-1$.

Proof. From $y_{1}(0)=y_{1}(T)$, there is a point $t_{1} \in[0, T]$ such that $y_{1}^{\prime}\left(t_{1}\right)=0$. Let $\omega_{1}(t)=y_{1}^{\prime}\left(t+t_{1}\right)$. Then, $\omega_{1}(0)=\omega_{1}(T)=0$. From $y_{2}(0)=y_{2}(T)$, there is a point $t_{2} \in[0, T]$ such that $y_{2}^{\prime}\left(t_{2}\right)=0$. Let $\omega_{2}(t)=y_{2}^{\prime}\left(t+t_{2}\right)$. Then, $\omega_{2}(0)=\omega_{2}(T)=0$. Continuing this way, we get from $y_{l-1}(0)=$ $y_{l-1}(T)$ a point $t_{l-1} \in[0, T]$ such that $y_{l-1}^{\prime}\left(t_{l-1}\right)=0$. Let $\omega_{l-1}(t)=y_{l-1}^{\prime}\left(t+t_{l-1}\right)$. Then, $\omega_{l-1}(0)=$ $\omega_{l-1}(T)=0$. From $y_{l}(t)=y_{l}(t+T)$, we have $\int_{0}^{T}\left(A y_{l}^{\prime}\right)(t) d t=\int_{0}^{T}\left(A y_{l}\right)^{\prime}(t) d t=\left.\left(A y_{l}\right)(t)\right|_{0} ^{T}=0$, so there is a point $t_{l} \in[0, T]$ such that $A y_{l}^{\prime}\left(t_{l}\right)=0$; hence, we have $\varphi_{p}\left(\left(A y_{l}^{\prime}\right)\left(t_{l}\right)\right)=0$. Let $\omega_{l}(t)=\varphi_{p}\left(\left(A y_{l}^{\prime}\right)\left(t+t_{l}\right)\right)=y_{l+1}\left(t+t_{l}\right)$. Then, $\omega_{l}(0)=\omega_{l}(T)=0$. Continuing this way, we get from $y_{n-1}(0)=y_{n-1}(T)$ that there is a point $t_{n-1} \in[0, T]$ such that $y_{n-1}^{\prime}\left(t_{n-1}\right)=0$. Let $\omega_{n-1}(t)=y_{n-1}^{\prime}\left(t+t_{n-1}\right)$. Then, $\omega_{n-1}(0)=\omega_{n-1}(T)=0$. By Lemma 2.2, we have

$$
\begin{aligned}
\int_{0}^{T}\left|y_{1}^{\prime}(t)\right|^{p} d t & =\int_{0}^{T}\left|\omega_{1}(t)\right|^{p} d t \\
& \leq\left(\frac{T}{\pi_{p}}\right)^{p} \int_{0}^{T}\left|\omega_{1}^{\prime}(t)\right|^{p} d t \\
& =\left(\frac{T}{\pi_{p}}\right)^{p} \int_{0}^{T}\left|y_{2}^{\prime}(t)\right|^{p} d t \\
& =\left(\frac{T}{\pi_{p}}\right)^{p} \int_{0}^{T}\left|\omega_{2}(t)\right|^{p} d t \\
& \leq\left(\frac{T}{\pi_{p}}\right)^{2 p} \int_{0}^{T}\left|\omega_{2}^{\prime}(t)\right|^{p} d t
\end{aligned}
$$


Boundary Value Problems

$$
\begin{aligned}
& \leq\left(\frac{T}{\pi_{p}}\right)^{p(l-1)} \int_{0}^{T}\left|\omega_{l-1}^{\prime}(t)\right|^{p} d t \\
& =\left(\frac{T}{\pi_{p}}\right)^{p(l-1)} \int_{0}^{T}\left|y_{l}^{\prime}(t)\right|^{p} d t .
\end{aligned}
$$

By Lemma 2.5 and Lemma 2.2, we have

$$
\begin{aligned}
\int_{0}^{T}\left|y_{l}^{\prime}(t)\right|^{p} d t & =\int_{0}^{T}\left|A^{-1} \varphi_{q}\left(y_{l+1}(t)\right)\right|^{p} d t \\
& \leq \frac{1}{|1-| c||^{p}} \int_{0}^{T}\left|\varphi_{q}\left(y_{l+1}(t)\right)\right|^{p} d t \\
& =\frac{1}{|1-| c||^{p}} \int_{0}^{T}\left|y_{l+1}(t)\right|^{p q-p} d t \\
& =\frac{1}{|1-| c||^{p}} \int_{0}^{T}\left|y_{l+1}(t)\right|^{q} d t \\
& =\frac{1}{\left.|1-| c\right|^{p}} \int_{0}^{T}\left|\omega_{l}(t)\right|^{q} d t \\
& \leq \frac{1}{\left.|1-| c\right|^{p}}\left(\frac{T}{\pi_{q}}\right)^{q} \int_{0}^{T}\left|\omega_{l}^{\prime}(t)\right|^{q} d t \\
& =\frac{1}{\left.|1-| c\right|^{p}}\left(\frac{T}{\pi_{q}}\right)^{q} \int_{0}^{T}\left|y_{l+2}(t)\right|^{q} d t \\
& \vdots \\
& \leq \frac{1}{|1-| c||^{p}}\left(\frac{T}{\pi_{q}}\right)^{q(n-l)} \int_{0}^{T}\left|\omega_{n-1}^{\prime}(t)\right|^{q} d t \\
& \frac{1}{|1-| c||^{p}}\left(\frac{T}{\pi_{q}}\right)^{q(n-l)} \int_{0}^{T}\left|y_{n}^{\prime}(t)\right|^{q} d t .
\end{aligned}
$$

Combining (2.35) and (2.36), we get

$$
\int_{0}^{T}\left|y_{1}^{\prime}(t)\right|^{p} d t \leq \frac{1}{|1-| c||^{p}}\left(\frac{T}{\pi_{p}}\right)^{p(l-1)}\left(\frac{T}{\pi_{q}}\right)^{q(n-l)} \int_{0}^{T}\left|y_{n}^{\prime}(t)\right|^{q} d t .
$$


Similarly, we get

$$
\int_{0}^{T}\left|y_{i}^{\prime}(t)\right|^{p} d t \leq \frac{1}{|1-| c||^{p}}\left(\frac{T}{\pi_{p}}\right)^{p(l-i)}\left(\frac{T}{\pi_{q}}\right)^{q(n-l)} \int_{0}^{T}\left|y_{n}^{\prime}(t)\right|^{q} d t .
$$

This completes the proof of Lemma 2.6.

Remark 2.7. In particular, if we take $p=2$, then $q=2$ and

$$
\pi_{p}=\pi_{q}=\pi_{2}=2 \int_{0}^{(2-1) / 2} \frac{d s}{\left(1-s^{2} /(2-1)\right)^{1 / 2}}=\frac{2 \pi(2-1)^{1 / 2}}{2 \sin (\pi / 2)}=\pi .
$$

In this case, (2.34) is transformed into

$$
\int_{0}^{T}\left|y_{i}(t)\right|^{2} d t \leq \frac{1}{|1-| c||^{2}}\left(\frac{T}{\pi}\right)^{2(n-i)} \int_{0}^{T}\left|y_{n}^{\prime}(t)\right|^{2} d t
$$

\section{Main Results}

For the sake of convenience, we list the following assumptions which will be used repeatedly in the sequel.

$\left(H_{1}\right)$ There exists a constant $D>0$ such that

$$
z_{1} F\left(t, z_{1}, z_{2}, \ldots, z_{l}\right)>0, \quad \forall\left(t, z_{1}, z_{2}, \ldots, z_{l}\right) \in[0, T] \times \mathbb{R}^{l}, \quad \text { with }\left|z_{1}\right|>D
$$

$\left(H_{2}\right)$ There exists a constant $M>0$ such that

$$
\left|F\left(t, z_{1}, z_{2}, \ldots, z_{l}\right)\right| \leq M, \quad \forall\left(t, z_{1}, z_{2}, \ldots, z_{l}\right) \in[0, T] \times \mathbb{R}^{l}
$$

$\left(H_{3}\right)$ There exist nonnegative constants $\alpha_{1}, \alpha_{2}, \ldots, \alpha_{l}, m$ such that

$$
\left|F\left(t, z_{1}, z_{2}, \ldots, z_{l}\right)\right| \leq \alpha_{1}\left|z_{1}\right|+\alpha_{2}\left|z_{2}\right|+\cdots+\alpha_{l}\left|z_{l}\right|+m, \quad \forall\left(t, z_{1}, z_{2}, \ldots, z_{l}\right) \in[0, T] \times \mathbb{R}^{l}
$$

$\left(H_{4}\right)$ There exist nonnegative constants $\gamma_{1}, \gamma_{2}, \ldots, \gamma_{n}$ such that

$$
\left|F\left(t, u_{1}, u_{2}, \ldots, u_{n}\right)-F\left(t, v_{1}, v_{2}, \ldots, v_{n}\right)\right| \leq \gamma_{1}\left|u_{1}-v_{1}\right|+\gamma_{2}\left|u_{2}-v_{2}\right|+\cdots+\gamma_{n}\left|u_{n}-v_{n}\right|
$$

for all $\left(t, u_{1}, u_{2}, \ldots, u_{n}\right),\left(t, v_{1}, v_{2}, \ldots, v_{n}\right) \in[0, T] \times \mathbb{R}^{n}$.

Theorem 3.1. If $\left(H_{1}\right)$ and $\left(H_{2}\right)$ hold, then (1.3) has at least one nonconstant T-periodic solution. 
Proof. Consider the equation

$$
L y=\lambda N y, \quad \lambda \in(0,1)
$$

Let $\Omega_{1}=\{y \in D(L): L y=\lambda N y, \lambda \in(0,1)\}$. If $y(t)=\left(y_{1}(t), y_{2}(t), \ldots, y_{n}(t)\right)^{\top} \in \Omega_{1}$, then

$$
\begin{aligned}
y_{1}^{\prime}(t) & =\lambda y_{2}(t), \\
y_{2}^{\prime}(t) & =\lambda y_{3}(t) \\
& \vdots \\
y_{l-1}^{\prime}(t) & =\lambda y_{l}(t) \\
y_{l}^{\prime}(t) & =\lambda \varphi_{q}\left(y_{l+1}(t)\right) \\
y_{l+1}^{\prime}(t) & =\lambda y_{l+2}(t) \\
& \vdots \\
y_{n-1}^{\prime}(t) & =\lambda y_{n}(t), \\
y_{n}^{\prime}(t) & =\lambda F\left(t, y_{1}(t), y_{2}(t), \ldots, y_{l}(t)\right) .
\end{aligned}
$$

We first claim that there exists a constant $\xi \in \mathbb{R}$ such that

$$
\left|y_{1}(\xi)\right| \leq D
$$

Integrating the last equation of (3.6) over $[0, T]$, we have

$$
\int_{0}^{T} F\left(t, y_{1}(t), y_{2}(t), \ldots, y_{l}(t)\right) d t=0
$$

By the continuity of $F$, there exists $\xi \in[0, T]$ such that

$$
F\left(\xi, y_{1}(\xi), \ldots, y_{l}(\xi)\right)=0
$$

From assumption $\left(H_{1}\right)$, we get (3.7). As a consequence, we have

$$
\left|y_{1}(t)\right|=\left|y_{1}(\xi)+\int_{\xi}^{t} y_{1}^{\prime}(s) d s\right| \leq D+\int_{0}^{T}\left|y_{1}^{\prime}(s)\right| d s
$$


On the other hand, multiplying both sides of the last equation of (3.6) by $y_{n}^{\prime}(t)$ and integrating over $[0, T]$, using assumption $\left(H_{2}\right)$, we have

$$
\begin{aligned}
\int_{0}^{T}\left|y_{n}^{\prime}(t)\right|^{2} d t & =\lambda \int_{0}^{T} F\left(t, y_{1}(t), y_{2}(t), \ldots, y_{l}(t)\right) y_{n}^{\prime}(t) d t \\
& \leq \int_{0}^{T}\left|F\left(t, y_{1}(t), y_{2}(t), \ldots, y_{l}(t)\right)\right|\left|y_{n}^{\prime}(t)\right| d t \\
& \leq M \int_{0}^{T}\left|y_{n}^{\prime}(t)\right| d t \\
& \leq M T^{1 / 2}\left(\int_{0}^{T}\left|y_{n}^{\prime}(t)\right|^{2} d t\right)^{1 / 2} .
\end{aligned}
$$

It is easy to see that there exists a constant $M_{n}^{\prime}>0$ (independent of $\lambda$ ) such that

$$
\int_{0}^{T}\left|y_{n}^{\prime}(t)\right|^{2} d t \leq M_{n}^{\prime}
$$

From $y_{n-1}(0)=y_{n-1}(T)$, there exists a point $t_{1} \in[0, T]$ such that $y_{n}\left(t_{1}\right)=0$. By Hölder's inequality, we have

$$
\left|y_{n}(t)\right| \leq \int_{0}^{T}\left|y_{n}^{\prime}(t)\right| d t \leq T^{1 / 2}\left(\int_{0}^{T}\left|y_{n}^{\prime}(t)\right|^{2} d t\right)^{1 / 2} \leq T^{1 / 2} M_{n}^{1^{1 / 2}}:=M_{n}
$$

From $y_{n-2}(0)=y_{n-2}(T)$, there exists a point $t_{2} \in[0, T]$ such that $y_{n-1}\left(t_{2}\right)=0$, and we have

$$
\left|y_{n-1}(t)\right| \leq \int_{0}^{T}\left|y_{n-1}^{\prime}(t)\right| d t=\int_{0}^{T}\left|\lambda y_{n}(t)\right| d t \leq \int_{0}^{T}\left|y_{n}(t)\right| d t \leq T M_{n}:=M_{n-1}
$$

Continuing this way for $y_{n-2}, \ldots, y_{l+1}$, we get

$$
\left|y_{l+1}(t)\right| \leq T M_{l+2}:=M_{l+1}
$$


Hence,

$$
\begin{aligned}
\left|y_{l}(t)\right| & \leq \int_{0}^{T}\left|y_{l}^{\prime}(t)\right| d t \leq \int_{0}^{T}\left|\lambda A^{-1} \varphi_{q}\left(y_{l+1}(t)\right)\right| d t \leq \frac{1}{|1-| c||} \int_{0}^{T}\left|y_{l+1}(t)\right|^{q-1} d t \\
& \leq \frac{1}{|1-| c||} T M_{l+1}^{q-1}:=M_{l} \\
\left|y_{l-1}(t)\right| & \leq T M_{l}:=M_{l-1}, \\
& \vdots \\
\left|y_{2}(t)\right| & \leq T M_{3}:=M_{2} .
\end{aligned}
$$

Meanwhile, from (3.10), we get

$$
\left|y_{1}(t)\right| \leq D+\int_{0}^{T}\left|y_{1}^{\prime}(t)\right| d t \leq D+T M_{2}:=M_{1}
$$

Let $M_{0}=\max \left\{M_{1}, M_{2}, \ldots, M_{n}\right\}$. Then, obviously $\left\|y_{1}\right\| \leq M_{0},\left\|y_{2}\right\| \leq M_{0}, \ldots$, and $\left\|y_{n}\right\| \leq M_{0}$. Let $\Omega_{2}=\{y \in \operatorname{Ker} L: N y \in \operatorname{Im} L\}$. If $y \in \Omega_{2}$, then $y \in \operatorname{Ker} L$, which means that $y=$ constant and $Q N y=0$. We see that

$$
\begin{gathered}
y_{2}=0, \\
y_{3}=0, \\
\vdots \\
y_{n}=0, \\
F\left(t, y_{1}, 0, \ldots, 0\right)=0 .
\end{gathered}
$$

So,

$$
\left|y_{1}\right| \leq D \leq M_{0}, \quad y_{2}=y_{3}=\cdots=y_{n}=0 \leq M_{0}
$$

Now take $\Omega=\left\{y=\left(y_{1}, y_{2}, \ldots, y_{n}\right)^{\top} \in X:\left\|y_{1}\right\|<M_{0}+1,\left\|y_{2}\right\|<M_{0}+1, \ldots,\left\|y_{n}\right\|<\right.$ $\left.M_{0}+1\right\}$. By the analysis above, it is easy to see that $\bar{\Omega}_{1} \subset \Omega, \bar{\Omega}_{2} \subset \Omega$, and conditions (1) and (2) of Lemma 2.1 are satisfied.

Next we show that condition (3) of Lemma 2.1 is also satisfied. Define an isomorphism $J: \operatorname{Im} Q \rightarrow$ Ker $L$ as follows:

$$
J\left(y_{1}, y_{2}, \ldots, y_{n}\right)^{\top}:=\left(y_{n}, y_{1}, \ldots, y_{n-1}\right)^{\top}
$$


Let $H(\mu, y)=\mu y+(1-\mu) J Q N y,(\mu, y) \in[0,1] \times \Omega$. Then, for all $(\mu, y) \in(0,1) \times(\partial \Omega \cap \operatorname{Ker} L)$,

$$
H(\mu, y)=\left(\begin{array}{c}
\mu y_{1}+\frac{1-\mu}{T} \int_{0}^{T} F\left(t, y_{1}, 0, \ldots, 0\right) d t \\
y_{2} \\
\vdots \\
A^{-1} \varphi_{q}\left(y_{l+1}\right) \\
\vdots \\
y_{n}
\end{array}\right)
$$

From $\left(H_{1}\right)$, it is obvious that $y H(\mu, y)>0$ for all $(\mu, y) \in(0,1) \times(\partial \Omega \cap \operatorname{Ker} L)$. Therefore,

$$
\begin{aligned}
\operatorname{deg}\{J Q N, \Omega \cap \operatorname{Ker} L, 0\} & =\operatorname{deg}\{H(0, y), \Omega \cap \operatorname{Ker} L, 0\} \\
& =\operatorname{deg}\{H(1, y), \Omega \cap \operatorname{Ker} L, 0\} \\
& =\operatorname{deg}\{I, \Omega \cap \operatorname{Ker} L, 0\} \neq 0,
\end{aligned}
$$

which means that condition (3) of Lemma 2.1 is also satisfied. By applying Lemma 2.1, we conclude that equation $L y=N y$ has a solution $y(t)^{*}=\left(y_{1}^{*}(t), y_{2}^{*}(t), \ldots, y_{n}^{*}(t)\right)^{\top}$ on $\bar{\Omega}$; that is, (1.3) has a $T$-periodic solution $y_{1}^{*}(t)$ with $\left\|y_{1}^{*}\right\|<M_{0}+1$.

Finally, observe that $y_{1}^{*}(t)$ is not constant. For, if $y_{1}^{*} \equiv a$ (constant), then from (1.3) we have $F(t, a, 0, \ldots, 0) \equiv 0$, which contradicts the assumption that $F(t, a, 0, \ldots, 0) \not \equiv 0$. The proof is complete.

Theorem 3.2. If $\left(\mathrm{H}_{1}\right)$ and $\left(\mathrm{H}_{3}\right)$ hold, then (1.3) has at least one nonconstant T-periodic solution if one of the following conditions holds:

(1) $p>2$,

(2) $p=2$ and $1 /|1-| c||\left[\left(\alpha_{1} T+\alpha_{2}\right)(T / \pi)^{l-1}+\alpha_{3}(T / \pi)^{l-2}+\cdots+\alpha_{l}(T / \pi)\right](T / \pi)^{n-l}<1$.

Proof. Let $\Omega_{1}$ be defined as in Theorem 3.1. If $y(t)=\left(y_{1}(t), y_{2}(t), \ldots, y_{n}(t)\right)^{\top} \in \Omega_{1}$, then from the proof of Theorem 3.1 we have

$$
\begin{gathered}
y_{n}^{\prime}(t)=\lambda F\left(t, y_{1}(t), y_{2}(t), \ldots, y_{l}(t)\right), \\
\left|y_{1}\right|_{0} \leq D+\int_{0}^{T}\left|y_{1}^{\prime}(s)\right| d s .
\end{gathered}
$$

We claim that $\left|y_{n}\right|_{0}$ is bounded. 
Boundary Value Problems

15

Multiplying both sides of (3.23) by $\varphi_{q}\left(y_{n}^{\prime}(t)\right)$ and integrating over $[0, T]$, by using assumption $\left(\mathrm{H}_{3}\right)$, we have

$$
\begin{aligned}
\int_{0}^{T}\left|y_{n}^{\prime}(t)\right|^{q} d t= & \lambda \int_{0}^{T} F\left(t, y_{1}(t), y_{2}(t), \ldots, y_{l}(t)\right) \varphi_{q}\left(y_{n}^{\prime}(t)\right) d t \\
\leq & \int_{0}^{T}\left|F\left(t, y_{1}(t), y_{2}(t), \ldots, y_{l}(t)\right)\right|\left|\varphi_{q}\left(y_{n}^{\prime}(t)\right)\right| d t \\
\leq & \alpha_{1} \int_{0}^{T}\left|y_{1}(t)\right|\left|\varphi_{q}\left(y_{n}^{\prime}(t)\right)\right| d t+\alpha_{2} \int_{0}^{T}\left|y_{2}(t)\right|\left|\varphi_{q}\left(y_{n}^{\prime}(t)\right)\right| d t \\
& +\cdots+\alpha_{l} \int_{0}^{T}\left|y_{l}(t)\right|\left|\varphi_{q}\left(y_{n}^{\prime}(t)\right)\right| d t+m \int_{0}^{T}\left|\varphi_{q}\left(y_{n}^{\prime}(t)\right)\right| d t \\
\leq & \alpha_{1}\left|y_{1}\right|_{0} \int_{0}^{T}\left|\varphi_{q}\left(y_{n}^{\prime}(t)\right)\right| d t+\alpha_{2} \int_{0}^{T}\left|y_{2}(t)\right|\left|\varphi_{q}\left(y_{n}^{\prime}(t)\right)\right| d t \\
& +\cdots+\alpha_{l} \int_{0}^{T}\left|y_{l}(t)\right|\left|\varphi_{q}\left(y_{n}^{\prime}(t)\right)\right| d t+m \int_{0}^{T}\left|\varphi_{q}\left(y_{n}^{\prime}(t)\right)\right| d t \\
\leq & \alpha_{1}\left(D+\int_{0}^{T}\left|y_{1}^{\prime}(t)\right| d t\right) \int_{0}^{T}\left|\varphi_{q}\left(y_{n}^{\prime}(t)\right)\right| d t+\alpha_{2} \int_{0}^{T}\left|y_{2}(t)\right|\left|\varphi_{q}\left(y_{n}^{\prime}(t)\right)\right| d t \\
& +\cdots+\alpha_{l} \int_{0}^{T}\left|y_{l}(t)\right|\left|\varphi_{q}\left(y_{n}^{\prime}(t)\right)\right| d t+m \int_{0}^{T}\left|\varphi_{q}\left(y_{n}^{\prime}(t)\right)\right| d t .
\end{aligned}
$$

Applying Hölder's inequality, we have

$$
\begin{aligned}
\int_{0}^{T}\left|y_{n}^{\prime}(t)\right|^{q} d t \leq & \alpha_{1}\left[D+T^{1 / p}\left(\int_{0}^{T}\left|y_{1}^{\prime}(t)\right|^{q} d t\right)^{1 / q}\right] T^{1 / q}\left(\int_{0}^{T}\left|\varphi_{q}\left(y_{n}^{\prime}(t)\right)\right|^{p} d t\right)^{1 / p} \\
& +\alpha_{2}\left(\int_{0}^{T}\left|y_{2}(t)\right|^{q} d t\right)^{1 / q}\left(\int_{0}^{T}\left|\varphi_{q}\left(y_{n}^{\prime}(t)\right)\right|^{p} d t\right)^{1 / p} \\
& +\cdots+\alpha_{l}\left(\int_{0}^{T}\left|y_{l}(t)\right|^{q} d t\right)^{1 / q}\left(\int_{0}^{T}\left|\varphi_{q}\left(y_{n}^{\prime}(t)\right)\right|^{p} d t\right)^{1 / p} \\
& +m T^{1 / q}\left(\int_{0}^{T}\left|\varphi_{q}\left(y_{n}^{\prime}(t)\right)\right|^{p} d t\right)^{1 / p} \\
\leq & \alpha_{1} T \cdot T^{(p-2) / q(p-1)}\left(\int_{0}^{T}\left|y_{1}^{\prime}(t)\right|^{p} d t\right)^{1 / q(p-1)}\left(\int_{0}^{T}\left|y_{n}^{\prime}(t)\right|^{q} d t\right)^{1 / p} \\
& +\alpha_{2} T^{(p-2) / q(p-1)}\left(\int_{0}^{T}\left|y_{2}(t)\right|^{p} d t\right)^{1 / q(p-1)}\left(\int_{0}^{T}\left|y_{n}^{\prime}(t)\right|^{q} d t\right)^{1 / p}+\cdots
\end{aligned}
$$


16

Boundary Value Problems

$$
\begin{aligned}
& +\alpha_{l} T^{(p-2) / q(p-1)}\left(\int_{0}^{T}\left|y_{l}(t)\right|^{p} d t\right)^{1 / q(p-1)}\left(\int_{0}^{T}\left|y_{n}^{\prime}(t)\right|^{q} d t\right)^{1 / p} \\
& +\left(\alpha_{1} D+m\right) T^{1 / q}\left(\int_{0}^{T}\left|y_{n}^{\prime}(t)\right|^{q} d t\right)^{1 / p} \\
& =\alpha_{1} T \cdot T^{(p-2) / p}\left(\int_{0}^{T}\left|y_{1}^{\prime}(t)\right|^{p} d t\right)^{1 / p}\left(\int_{0}^{T}\left|y_{n}^{\prime}(t)\right|^{q} d t\right)^{1 / p} \\
& +\alpha_{2} T^{(p-2) / p}\left(\int_{0}^{T}\left|y_{2}(t)\right|^{p} d t\right)^{1 / p}\left(\int_{0}^{T}\left|y_{n}^{\prime}(t)\right|^{q} d t\right)^{1 / p}+ \\
& \cdots+\alpha_{l} T^{(p-2) / p}\left(\int_{0}^{T}\left|y_{l}(t)\right|^{p} d t\right)^{1 / p}\left(\int_{0}^{T}\left|y_{n}^{\prime}(t)\right|^{q} d t\right)^{1 / p} \\
& +\left(\alpha_{1} D+m\right) T^{1 / q}\left(\int_{0}^{T}\left|y_{n}^{\prime}(t)\right|^{q} d t\right)^{1 / p} .
\end{aligned}
$$

Applying Lemma 2.6 and (3.26), we have

$$
\begin{aligned}
\int_{0}^{T}\left|y_{n}^{\prime}(t)\right|^{q} d t \leq & \alpha_{1} T \cdot T^{(p-2) / p} \frac{1}{|1-| c||}\left(\frac{T}{\pi_{p}}\right)^{l-1}\left(\frac{T}{\pi_{q}}\right)^{q(n-l) / p}\left(\int_{0}^{T}\left|y_{n}^{\prime}(t)\right|^{q} d t\right)^{2 / p} \\
& +\alpha_{2} T^{(p-2) / p} \frac{1}{|1-| c||}\left(\frac{T}{\pi_{p}}\right)^{l-1}\left(\frac{T}{\pi_{q}}\right)^{q(n-l) / p}\left(\int_{0}^{T}\left|y_{n}^{\prime}(t)\right|^{q} d t\right)^{2 / p}+ \\
& \cdots+\alpha_{l} T^{(p-2) / p} \frac{1}{|1-| c||}\left(\frac{T}{\pi_{p}}\right)\left(\frac{T}{\pi_{q}}\right)^{q(n-l) / p}\left(\int_{0}^{T}\left|y_{n}^{\prime}(t)\right|^{q} d t\right)^{2 / p} \\
& +\left(\alpha_{1} D+m\right) T^{1 / q}\left(\int_{0}^{T}\left|y_{n}^{\prime}(t)\right|^{q} d t\right)^{1 / p} \\
\leq & \frac{1}{|1-| c||}\left[\left(\alpha_{1} T+\alpha_{2}\right)\left(\frac{T}{\pi_{p}}\right)^{l-1}+\alpha_{3}\left(\frac{T}{\pi_{p}}\right)^{l-2}+\cdots+\alpha_{l}\left(\frac{T}{\pi_{p}}\right)\right] \\
& \times T^{(p-2) / p}\left(\frac{T}{\pi_{q}}\right)^{q(n-l) / p} \cdot\left(\int_{0}^{T}\left|y_{n}^{\prime}(t)\right|^{q} d t\right)^{2 / p} \\
& +\left(\alpha_{1} D+m\right) T^{1 / q}\left(\int_{0}^{T}\left|y_{n}^{\prime}(t)\right|^{q} d t\right)^{1 / p} .
\end{aligned}
$$


Case 1. If $p=2$ and $1 /|1-| c||\left[\left(\alpha_{1} T+\alpha_{2}\right)(T / \pi)^{l-1}+\alpha_{3}(T / \pi)^{l-2}+\cdots+\alpha_{l}(T / \pi)\right](T / \pi)^{n-l}<1$, then it is easy to see that there exists a constant $M_{n}^{\prime}>0$ (independent of $\lambda$ ) such that

$$
\int_{0}^{T}\left|y_{n}^{\prime}(t)\right|^{q} d t \leq M_{n}^{\prime}
$$

Case 2. If $p>2$, then it is easy to see that there exists a constant $M_{n}^{\prime}>0$ (independent of $\lambda$ ) such that

$$
\int_{0}^{T}\left|y_{n}^{\prime}(t)\right|^{q} d t \leq M_{n}^{\prime}
$$

From $y_{n-1}(0)=y_{n-1}(T)$, there exists a point $t_{1} \in[0, T]$ such that $y_{n}\left(t_{1}\right)=0$. By Hölder's inequality, we have

$$
\left|y_{n}(t)\right| \leq \int_{0}^{T}\left|y_{n}^{\prime}(t)\right| d t \leq T^{1 / p}\left(\int_{0}^{T}\left|y_{n}^{\prime}(t)\right|^{q} d t\right)^{1 / q} \leq T^{1 / p} M_{n}^{\prime 1 / q}:=M_{n}
$$

This proves the claim, and the rest of the proof of the theorem is identical to that of Theorem 3.1.

Remark 3.3. If (1.3) takes the form

$$
\left(\varphi_{p}(x(t)-c x(t-\sigma))^{(l)}\right)^{(n-l)}=F\left(t, x(t), x^{\prime}(t), \ldots, x^{(l-1)}(t)\right)+e(t)
$$

where $e(t) \in C(\mathbb{R}, \mathbb{R}), e(t+T)=e(t)$ and $\int_{0}^{T} e(t) d t=0$, then the results of Theorems 3.1 and 3.2 still hold.

Remark 3.4. If $p=2$, then (1.3) is transformed into

$$
(x(t)-c x(t-\sigma))^{(n)}=F\left(t, x(t), x^{\prime}(t), \ldots, x^{(n-1)}(t)\right),
$$

and the results of Theorems 3.1 and 3.2 still hold.

Next, we study the Lyapunov stability of the periodic solutions of (3.32).

Theorem 3.5. Assume that $\left(\mathrm{H}_{4}\right)$ holds. Then every T-periodic solution of (3.32) is Lyapunov stable.

Proof. Let

$$
z_{1}(t)=x(t), \quad z_{2}(t)=x^{\prime}(t), \ldots, z_{n}(t)=x^{(n-1)}(t) .
$$


Then, system (3.32) is transformed into

$$
\begin{gathered}
z_{1}^{\prime}(t)=z_{2}(t), \\
z_{2}^{\prime}(t)=z_{3}(t), \\
\vdots \\
z_{n}^{\prime}(t)=A^{-1} F\left(t, z_{1}(t), z_{2}(t), \ldots, z_{n}(t)\right) .
\end{gathered}
$$

Suppose now that $z^{*}(t)=\left(z_{1}^{*}(t), z_{2}^{*}(t), \ldots, z_{n}^{*}(t)\right)^{\top}$ is a $T$-periodic solution of (3.34). Let $z(t)=\left(z_{1}(t), z_{2}(t), \ldots, z_{n}(t)\right)^{\top}$ be any arbitrary solution of (3.34). For any $k=1, \ldots, n$, write $w_{k}(t)=z_{k}(t)-z_{k}^{*}(t)$. Then, it follows from (3.34) that

$$
\begin{gathered}
w_{1}^{\prime}(t)=w_{2}(t), \\
w_{2}^{\prime}(t)=w_{3}(t), \\
\vdots \\
w_{n}^{\prime}(t)=A^{-1}\left(F\left(t, z_{1}(t), z_{2}(t), \ldots, z_{n}(t)\right)-F\left(t, z_{1}^{*}(t), z_{2}^{*}(t), \ldots, z_{n}^{*}(t)\right)\right),
\end{gathered}
$$

and so

$$
\begin{gathered}
\left|w_{1}^{\prime}(t)\right|=\left|w_{2}(t)\right|, \\
\left|w_{2}^{\prime}(t)\right|=\left|w_{3}(t)\right|, \\
\vdots \\
\left|w_{n}^{\prime}(t)\right|=\left|A^{-1}\left(F\left(t, z_{1}(t), z_{2}(t), \ldots, z_{n}(t)\right)-F\left(t, z_{1}^{*}(t), z_{2}^{*}(t), \ldots, z_{n}^{*}(t)\right)\right)\right| .
\end{gathered}
$$

Let $u_{k}^{(l)}(t)=\left|w_{k}^{(l)}(t)\right|, l=0,1, k=1,2, \ldots, n$. Then,

$$
\begin{gathered}
u_{1}^{\prime}(t)=u_{2}(t), \\
u_{2}^{\prime}(t)=u_{3}(t), \\
\vdots \\
u_{n}^{\prime}(t)=\left|A^{-1}\left(F\left(t, z_{1}(t), z_{2}(t), \ldots, z_{n}(t)\right)-F\left(t, z_{1}^{*}(t), z_{2}^{*}(t), \ldots, z_{n}^{*}(t)\right)\right)\right| .
\end{gathered}
$$

Take $\beta=\max \left\{\gamma_{1} /|1-| c||, \gamma_{2} /|1-| c||+1, \ldots, \gamma_{n} /|1-| c||+1\right\}+1$, and define a function $V(\cdot)$ by

$$
V\left(t, u_{1}, \ldots, u_{n}\right):=e^{-\beta t} \sum_{k=1}^{n} u_{k}(t)
$$


Let $U\left(u_{1}, \ldots, u_{n}\right)=\sum_{k=1}^{n} y_{k}(t)$. It is obvious that $V\left(t, u_{1}, \ldots, u_{n}\right)>0$ and $V\left(t, u_{1}, \ldots, u_{n}\right) \geq$ $U\left(u_{1}, \ldots, u_{n}\right)>0$. From $\left(H_{4}\right)$ and Lemma 2.3, we get

$$
\begin{aligned}
& \dot{V}\left(t, u_{1}, \ldots, u_{n}\right)=-\beta e^{-\beta t}\left(\sum_{k=1}^{n} u_{k}(t)\right)+e^{-\beta t}\left(u_{2}(t)+\cdots+u_{n}(t)\right) \\
& +e^{-\beta t}\left|A^{-1}\left(F\left(t, z_{1}(t), z_{2}(t), \ldots, z_{n}(t)\right)-F\left(t, z_{1}^{*}(t), z_{2}^{*}(t), \ldots, z_{n}^{*}(t)\right)\right)\right| \\
& \leq-\beta e^{-\beta t}\left(\sum_{k=1}^{n} u_{k}(t)\right)+e^{-\beta t}\left(u_{2}(t)+\cdots+u_{n}(t)\right) \\
& +\frac{e^{-\beta t}}{|1-| c||}\left|\left(F\left(t, z_{1}(t), z_{2}(t), \ldots, z_{n}(t)\right)-F\left(t, z_{1}^{*}(t), z_{2}^{*}(t), \ldots, z_{n}^{*}(t)\right)\right)\right| \\
& \leq-\beta e^{-\beta t}\left(\sum_{k=1}^{n} u_{k}(t)\right)+e^{-\beta t}\left(u_{2}(t)+\cdots+u_{n}(t)\right) \\
& +\frac{e^{-\beta t}}{|1-| c||}\left(\gamma_{1}\left|z_{1}(t)-z_{1}^{*}(t)\right|+\cdots+\gamma_{n}\left|z_{n}(t)-z_{n}^{*}(t)\right|\right) \\
& =-\beta e^{-\beta t}\left(\sum_{k=1}^{n} u_{k}(t)\right)+e^{-\beta t}\left(u_{2}(t)+\cdots+u_{n}(t)\right) \\
& +\frac{e^{-\beta t}}{|1-| c||}\left(\gamma_{1}\left|w_{1}(t)\right|+\cdots+\gamma_{n}\left|w_{n}(t)\right|\right) \\
& =-\beta e^{-\beta t}\left(\sum_{k=1}^{n} u_{k}(t)\right)+e^{-\beta t}\left(u_{2}(t)+\cdots+u_{n}(t)\right) \\
& +\frac{e^{-\beta t}}{|1-| c||}\left(\gamma_{1} u_{1}(t)+\cdots+\gamma_{n} u_{n}(t)\right) \\
& =\left(-\beta+\frac{\gamma_{1}}{|1-| c||}\right) u_{1}(t) e^{-\beta t}+\sum_{k=2}^{n}\left(-\beta+1+\frac{\gamma_{k}}{|1-| c||}\right) u_{k}(t) e^{-\beta t} \\
& <0 \text {. }
\end{aligned}
$$

Hence, $V$ is a Lyapunov function for nonautonomous (3.32) (see [15, page 50]), and so the $T$-periodic solution $z^{*}$ of (3.32) is Lyapunov stable.

Finally, we present an example to illustrate our result.

Example 3.6. Consider the $n$-order delay differential equation

$$
\left(\varphi_{p}(x(t)-3 x(t-\sigma))^{(4)}\right)^{(n-4)}=\frac{1}{3 \pi} x(t)+\frac{1}{8} \sin x^{\prime}(t)+\frac{1}{8} \cos x^{\prime \prime}(t) \sin 2 t+\frac{1}{8} \sin x^{\prime \prime \prime}(t) .
$$


Here $p$ is a constant with $p \geq 2$. Comparing with (1.3), we have $c=3$ and

$$
F\left(t, z_{1}, z_{2}, z_{3}, z_{4}\right)=\frac{1}{3 \pi} z_{1}+\frac{1}{8} \sin z_{2}+\frac{1}{8} \cos z_{3} \sin 2 t+\frac{1}{8} \sin z_{4} .
$$

Observe that $F$ has period $T=\pi$ and satisfies

$$
F(t, a, 0,0,0)=\frac{1}{3 \pi} a+\frac{1}{8} \sin 2 t \not \equiv 0, \quad \forall a \in \mathbb{R} .
$$

Pick $D=3 \pi$. Then,

$$
\begin{aligned}
\left|z_{1} F\left(t, z_{1}, z_{2}, z_{3}, z_{4}\right)\right| & =\left|z_{1}\right| \cdot\left|\frac{1}{3 \pi} z_{1}+\frac{1}{8} \sin z_{2}+\frac{1}{8} \cos z_{3} \sin 2 t+\frac{1}{8} \sin z_{4}\right| \\
& \geq\left|z_{1}\right| \cdot\left|\frac{1}{3 \pi}\right| z_{1}|-| \frac{1}{8} \sin z_{2}+\frac{1}{8} \cos z_{3} \sin 2 t+\frac{1}{8} \sin z_{4}|| \\
& \geq 3 \pi \cdot\left[1-\frac{3}{8}\right] \\
& >0
\end{aligned}
$$

for all $\left(t, z_{1}, z_{2}, z_{3}, z_{4}\right) \in[0, T] \times \mathbb{R}^{l}$ with $\left|z_{1}\right|>D=3 \pi$. Hence, $\left(H_{1}\right)$ holds. On the other hand, since

$$
\begin{aligned}
\left|F\left(t, z_{1}, z_{2}, z_{3}, z_{4}\right)\right| & \leq \frac{1}{3 \pi}\left|z_{1}\right|+\left|\frac{1}{8} \sin z_{2}+\frac{1}{8} \cos z_{3} \sin 2 t+\frac{1}{8} \sin z_{4}\right| \\
& <\frac{1}{3 \pi}\left|z_{1}\right|+1
\end{aligned}
$$

assumption $\left(H_{3}\right)$ holds with $\alpha_{1}=1 / 3 \pi, \alpha_{2}=0, \alpha_{3}=0, \alpha_{4}=0$, and $m=1$.

Case 1. If $p>2$, then by (1) of Theorem 3.2, (3.40) has at least one nonconstant $\pi$-periodic solution.

Case 2. If $p=2$, then

$$
\begin{aligned}
\frac{1}{|1-| c||} & {\left[\left(\alpha_{1} T+\alpha_{2}\right)\left(\frac{T}{\pi}\right)^{3}+\alpha_{3}\left(\frac{T}{\pi}\right)^{2}+\alpha_{4}\left(\frac{T}{\pi}\right)\right]\left(\frac{T}{\pi}\right)^{n-4} } \\
= & \frac{1}{2} \times\left[\left(\frac{1}{3 \pi} \times \pi+0\right) \times 1+0 \times 1+0 \times 1\right] \times 1 \\
= & \frac{1}{2} \times \frac{1}{3}<1 .
\end{aligned}
$$

So by (2) of Theorem 3.2, (3.40) has at least one nonconstant $\pi$-periodic solution. 


\section{Acknowledgments}

This paper is partially supported by the National Natural Science Foundation of China (10971202), and the Research Grant Council of Hong Kong SAR, China (project no. HKU7016/07P).

\section{References}

[1] S. Lu and W. Ge, "On the existence of periodic solutions for neutral functional differential equation," Nonlinear Analysis: Theory, Methods \& Applications, vol. 54, no. 7, pp. 1285-1306, 2003.

[2] S. Lu, "Existence of periodic solutions for a $p$-Laplacian neutral functional differential equation," Nonlinear Analysis: Theory, Methods E Applications, vol. 70, no. 1, pp. 231-243, 2009.

[3] S. Lu, W. Ge, and Z. Zheng, "Periodic solutions to neutral differential equation with deviating arguments," Applied Mathematics and Computation, vol. 152, no. 1, pp. 17-27, 2004.

[4] S. Peng, "Periodic solutions for $p$-Laplacian neutral Rayleigh equation with a deviating argument," Nonlinear Analysis: Theory, Methods \& Applications, vol. 69, no. 5-6, pp. 1675-1685, 2008.

[5] J. Ren and Z. Cheng, "Periodic solutions for generalized high-order neutral differential equation in the critical case," Nonlinear Analysis: Theory, Methods \& Applications, vol. 71, no. 12, pp. 6182-6193, 2009.

[6] J. Shen and R. Liang, "Periodic solutions for a kind of second order neutral functional differential equations," Applied Mathematics and Computation, vol. 190, no. 2, pp. 1394-1401, 2007.

[7] Q. Wang and B. Dai, "Three periodic solutions of nonlinear neutral functional differential equations," Nonlinear Analysis: Real World Applications, vol. 9, no. 3, pp. 977-984, 2008.

[8] K. Wang and S. Lu, "On the existence of periodic solutions for a kind of high-order neutral functional differential equation," Journal of Mathematical Analysis and Applications, vol. 326, no. 2, pp. 1161-1173, 2007.

[9] J. Wu and Z. Wang, "Two periodic solutions of second-order neutral functional differential equations," Journal of Mathematical Analysis and Applications, vol. 329, no. 1, pp. 677-689, 2007.

[10] M. R. Zhang, "Periodic solutions of linear and quasilinear neutral functional-differential equations," Journal of Mathematical Analysis and Applications, vol. 189, no. 2, pp. 378-392, 1995.

[11] Y. Zhu and S. Lu, "Periodic solutions for $p$-Laplacian neutral functional differential equation with deviating arguments," Journal of Mathematical Analysis and Applications, vol. 325, no. 1, pp. 377-385, 2007.

[12] R. E. Gaines and J. L. Mawhin, Coincidence Degree, and Nonlinear Differential Equations, Lecture Notes in Mathematics, vol. 568, Springer, Berlin, Germany, 1977.

[13] M. Zhang, "Nonuniform nonresonance at the first eigenvalue of the $p$-Laplacian," Nonlinear Analysis: Theory, Methods \& Applications, vol. 29, no. 1, pp. 41-51, 1997.

[14] J. K. Hale, Theory of Functional Differential Equations, Springer, New York, NY, USA, 1977.

[15] P. Glendinning, Stability, Instability and Chaos: An Introduction to the Theory of Nonlinear Differential Equations, Cambridge Texts in Applied Mathematics, Cambridge University Press, Cambridge, UK, 1994. 